\title{
EXPOSICIÓN FETAL A BISFENOL A: PRESENCIA DE BISFENOL A EN ORINA DE MUJERES GESTANTES ASISTIDAS EN UN HOSPITAL DE LA CIUDAD DE BUENOS AIRES. AÑO 2013.
}

\section{FETAL EXPOSURE TO BISPHENOL A: PRESENCE OF BISPHENOL A IN URINE OF PREGNANT WOMEN ASSISTED IN A HOSPITAL IN THE CITY OF BUENOS AIRES. YEAR 2013.}

\author{
Analía Ferloni ${ }^{1,3}$, Natalia Pereiro ${ }^{1}$, Martín Cruz ${ }^{1}$, Soledad Aragone ${ }^{1}$, Paula Kandel Gambarte ${ }^{2}$, Valeria Aliperti ${ }^{1}$, \\ Julia Constanza Saulino ${ }^{1}$, Olivia Cambiaso ${ }^{1}$, Marcela Liliam Vázquez ${ }^{1}$, Mariana Laura Méndez ${ }^{1}$, Claudio Fernando \\ Moix ${ }^{1}$, Flavia Alejandra Vidal', Raúl Uicich ${ }^{1}$, Lucas Otaño ${ }^{1}$, Silvana Figar ${ }^{1}$, María Isabel Giménez ${ }^{1}$, Carlos Gustavo \\ Wahren".
}

1 Hospital Italiano de Buenos Aires

2 Universidad de Buenos Aires.

3 Email de contacto: analia.ferloni@hospitalitaliano.org.ar

\section{Conceptos clave:}

El Bisfenol A (BPA) es un químico utilizado a nivel mundial en la fabricación de envases de alimentos y bebidas. Se comporta como disruptor endócrino, actuando a concentraciones séricas bajas pudiendo alterar la función hormonal, modificando la expresión del ADN pudiendo interferir en los momentos críticos del desarrollo embrionario.

En todos los estudios publicados, se obtuvo como resultado una exposición generalizada al BPA. En nuestro país no se han desarrollado aún estudios que evalúen la presencia de BPA en poblaciones humanas por lo que la misma se desconoce.

En Argentina, se reguló el uso de BPA en la fabricación y comercialización de mamaderas.

Debido a la exposición generalizada al BPA, y que no se cuenta con suficiente evidencia de efectos a largo plazo de la exposición transplacentaria, este trabajo puede aportar conocimiento sobre la exposición de nuestra población y aportar datos basales para futuros estudios de riesgo.

Recibido: 2018-12-21 Aceptado: 2019-02-23 DOI: http://dx.doi.org/10.31053/1853.0605.v76.n2.22806 (c) (i) (8)

(C) Universidad Nacional de Córdoba

\section{Resumen:}

Introducción: El Bisfenol A (BPA) es un disruptor endócrino (DE) posiblemente vinculado a eventos adversos reproductivos y del desarrollo neurológico que se utiliza como aditivo en envases plásticos de alimentos y bebidas. En la población argentina no existen datos sobre niveles de BPA en orina. El objetivo del estudio fue medir la presencia de BPA en orina de embarazadas.

Métodos: Estudio transversal. Detección de BPA en orina de embarazadas atendidas en el Hospital Italiano de Buenos Aires en el año 2013. La técnica utilizada fue cromatografía gaseosa asociada a espectrometría de masa con un límite de cuantificación de $2 \mathrm{ng} / \mathrm{ml}$ y de detección de $0,8 \mathrm{ng} / \mathrm{ml}$. Se describe la proporción de mujeres con exposición al BPA con su intervalo de confianza del 95\% (IC95\%).

Resultados: se analizaron 149 muestras de orina de embarazadas de edad promedio 32 años (Desvío estándar 5,3), el 81\% (IC95\% 73,9-86,9) trabaja y el 83,7\% (IC95\% 76,789,1 ) posee nivel universitario y/o posgrado. El 66,4\% (IC95\% 58,2-74,0) de las muestras urinarias presentaron BPA cuantificable, siendo la mediana de BPA de 4,3 ng / mg creatinina (Rango Intercuartilo 3,2-6,7).

Conclusiones: Este estudio muestra que la exposición a BPA durante el proceso de formación embrionaria en un sector de la población de Argentina, es generalizada. En Argentina solo está regulado el uso de este DE en mamaderas.

Palabras claves: bisfenol a glicidil metacrilato; mujeres embarazadas; orina; disruptores endocrinos; epidemiología; salud ambiental.

\section{Abstract:}

Introduction: Bisphenol A (BPA) is an endocrine disruptor (ED) possibly linked to adverse reproductive and neurodevelopmental effects that is used as an additive in food and beverage containers. There are no data of BPA levels in the Argentine population. The objective of this study was to measure BPA in the urine of pregnant women.

Methods: Cross-sectional study. Detection of BPA in pregnant women of the Hospital Italiano de Buenos Aires in 2013, using gas chromatography and mass spectrometry with a limit of quantification of $2 \mathrm{ng} / \mathrm{ml}$ and detection of $0.8 \mathrm{ng} / \mathrm{ml}$. The proportion of women exposed to BPA within the 95\% confidence interval (IC 95\%) is described.

Results: 149 pregnant women, average age 32 years (Standard Deviation 5.3), 81\% $(95 \% \mathrm{Cl} 73.9-86.9)$ workers and $83.7 \%(95 \% \mathrm{Cl} 76.7-89.1)$ have university or higher education, $66.4 \%(95 \% \mathrm{Cl} 58-74.0)$ of the urinary samples were quantifiable, with a median BPA of $4.3 \mathrm{ng} / \mathrm{mg} \mathrm{Cr}$ (RI 3.2-6.7).

Conclusions: This study shows that exposure to BPA during the process of embryonic development in a sector of the population of Argentina is widespread. In Argentina, the use of this ED is only regulated in baby bottles.

Keywords: bisphenol a glycidyl methacrylate; pregnant women; urine; endocrine disruptors; epidemiology; environmental health. 


\section{Introducción}

Los disruptores endocrinos (DEs) son sustancias exógenas al organismo que alteran la función del sistema endócrino, pudiendo provocar efectos adversos en la salud de un organismo y/o de su descendencia ${ }^{(1-2)}$. Una particularidad de los DEs es que actúan a concentraciones séricas extremadamente bajas de la misma manera que lo hacen las hormonas naturales, por lo que los efectos tóxicos pueden presentarse, incluso a niveles picomolares ${ }^{(3)}$.

Entre los DEs utilizados cotidianamente se encuentran pesticidas, plastificantes, compuestos clorados, ftalatos, estireno, polibromodifeniléteres (PBDE), polibromuros de bifenilo (PBB) y el bisfenol-A (BPA) ${ }^{(4)}$

El BPA es uno de los químicos de mayor volumen de producción a nivel mundial utilizado principalmente en la fabricación de plásticos de policarbonato y resinas epoxi ${ }^{(5)}$, como pueden ser envases, recubrimientos de latas de conservas, botellas de plástico, CDs, papel térmico, anteojos de sol, instrumental médico u odontológico, etc. ${ }^{(6)}$.

Se ha demostrado que la exposición más frecuente de la población general al BPA es a través de la ingesta de alimentos y bebidas, siendo la comida en conserva o enlatada la principal fuente ${ }^{(7)}$.

EI BPA al entrar en contacto con alimentos $y / 0$ bebidas, puede migrar a los mismos. Dicha migración está influenciada por distintos factores como: acidez de los alimentos, cambios de temperatura producidos durante el proceso de transporte, almacenamiento, esterilización de los alimentos enlatados así como el calentamiento de leche en mamaderas ${ }^{(8)}$.

Una vez que el BPA ingresa al organismo, su metabolización se produce en el hígado mediante glucuronización y sulfonación y es excretado por orina. Su metabolito principal, el BPA-glucurónido pierde su efecto ${ }^{(9)}$.

EI BPA traspasa la barrera placentaria y los niveles en sangre fetal pueden superar los presentes en sangre materna ${ }^{(10)}$. Las concentraciones intraútero pueden alcanzar rangos hasta 10 veces mayores que los descritos en adultos ${ }^{(10)}$. La exposición fetal a distintos compuestos químicos, a través de la placenta, está dada tanto por la carga previa de los compuestos persistentes almacenados en el tejido adiposo materno y movilizado durante el embarazo así como por la exposición de la madre durante el embarazo ${ }^{(11)}$.

El mecanismo de acción del BPA varía según dosis, tipo y magnitud de la exposición, así como del tejido y la etapa del desarrollo fetal en que se produce la misma ${ }^{(12)}$. Actúa principalmente como un potente agonista de receptores estrogénicos (RE) mimetizando la acción hormonal ${ }^{(13)}$, pudiendo alterar respuestas celulares a dosis muy bajas por su capacidad de unirse a los RE con gran afinidad ${ }^{(14)}$. Otro mecanismo de acción del BPA son las alteraciones epigenéticas $^{(15)}$, que pueden evidenciar sus consecuencias en la progenie en períodos muy posteriores dificultando la asociación temporal con la exposición durante la etapa embrionaria.

Este efecto endocrino del BPA y su impacto sobre la descendencia fue evidenciado inicialmente en $\operatorname{ratas}^{(16)}$ y luego en seres humanos $^{(17)}$. En la vida intrauterina y en las primeras etapas del desarrollo, las enzimas que metabolizan el BPA tienen menor actividad, lo que aumenta la concentración de BPA circulante y expone a esa población a una mayor acción estrogénica. También se ha demostrado que los fetos, los bebés y los niños muestran mayor susceptibilidad que los adultos al BPA ${ }^{(18)}$.

Dentro de los efectos del BPA ${ }^{(19)}$ se observó incremento en el peso de los recién nacidos cuyas madres tenían valores elevados de BPA en orina ${ }^{(20)}$, efectos reproductivos ${ }^{(21)}$, adelanto de la menarca, reducción de la memoria y capacidad de aprendizaje ${ }^{(22)}$, y alteraciones en el desarrollo del aparato genitourinario como por ejemplo hipospadias y disminución de la distancia ano-genital ${ }^{(20)}$. Las poblaciones foliculares de los ovarios también son afectadas por el BPA. Mujeres con altas concentraciones de BPA en sangre y orina muestran una respuesta deficitaria a tratamientos de estimulación exógena en protocolos de fertilización in vitro ${ }^{(23)}$.

La exposición a BPA en distintas poblaciones es generalizada. Esto fue confirmado tanto por La Encuesta de Salud y Nutrición Nacional de EE.UU. (National Health and Nutrition Examination Survey,
NHANES) publicada en el año 2008, como por la encuesta Health Canada realizada en el año 2010, en las que se midió concentraciones urinarias de $\mathrm{BPA}^{(24)}$. En todos los casos se encontró que entre el 90 y el $95 \%$ de la población está expuesta a esta sustancia ${ }^{(23,25)}$.

En nuestro país no encontramos datos sobre esta exposición, lo que motivó a realizar una medición de BPA al equipo del Programa de Investigación en Salud Ambiental (PISA) del Hospital Italiano de Buenos Aires. Este programa se conformó en el año 2012 y de manera transdisciplinaria trabaja desde entonces en la prevención y producción de evidencia sobre distintas exposiciones ambientales. En este trabajo se informa la presencia de BPA en orina de embarazadas como expresión de la medición indirecta de exposición fetal.

\section{Objetivo}

Determinar la presencia de BPA en orina de mujeres embarazadas atendidas en el Hospital Italiano de Buenos Aires.

\section{Materiales y métodos}

Diseño: Estudio descriptivo de corte transversal, en orina de mujeres embarazadas que se atendieron en el servicio de obstetricia del Hospital Italiano de Buenos Aires (HIBA).

Ámbito: El HIBA es un hospital universitario que posee una red asistencial de alta complejidad, que brinda servicios médicos a más de 150.000 personas. Cuenta con dos hospitales principales y 19 centros de atención distribuidos en diferentes barrios de la Ciudad Autónoma de Buenos Aires y de conurbano bonaerense. El servicio de obstetricia atiende a gestantes con coberturas de salud diversas, y realiza un promedio de 200 partos mensuales.

Período de estudio: Agosto - septiembre del año 2013.

Población: La población atendida en el HIBA cuenta con cobertura de salud prepaga u obra social y pertenece, en su mayoría, a clase media urbana. Residen en Ciudad Autónoma de Buenos Aires y Gran Buenos Aires.

Se incluyeron mujeres embarazadas de 18 años o más, con una edad gestacional entre 11 y 16 semanas, que realizaban los controles obstétricos en el HIBA.

Las pacientes fueron invitadas a participar del estudio en la sala de espera mientras aguardaban realizarse la ecografía de translucencia nucal. En el HIBA esta práctica es de rutina para todas las gestantes entre la $11^{6}$ y $13^{6}$ semanas de embarazo. Luego de aceptar participar mediante la firma del Consentimiento Informado, se completó una ficha con datos sociodemográficos y se les facilitó un frasco de urocultivo para la toma de muestra de orina. El total de las muestras fueron anonimizadas y se les otorgó un valor numérico consecutivo.

Medición de BPA: La recolección de la muestra de orina se realizó en envases de polipropileno libres de $\mathrm{BPA}^{(26)}$ y fueron almacenadas a $-80{ }^{\circ} \mathrm{C}$ hasta su análisis ${ }^{(26)}$. La medición de las concentraciones urinarias de BPA se efectúo en el laboratorio del HIBA mediante cromatografía gaseosa acoplada a un detector de masa de simple cuádruple (GC/MS) en modo SIM, empleando material de vidrio, consumibles y reactivos libres de BPA. El método bio analítico para la cuantificación de BPA libre en orina consiste en un tratamiento enzimático previo con Beta glucuronidasa, posterior extracción de BPA con acetato de etilo. El extracto orgánico posteriormente es evaporado a sequedad con corriente de nitrógeno y derivatizado con NA-bis (trimetilsilil) trifluoracetamida $(\mathrm{BSTFA})^{(27)}$

Determinación de presencia de BPA en orina $y$ definición de la variable BPA: La concentración de BPA se expresó como $\mathrm{ng} / \mathrm{ml}$ y $\mathrm{ng} / \mathrm{mg}$ de creatinina. 
Las muestras se clasificaron en categorías según los límites de detección y cuantificación establecidos por nuestro laboratorio (Tabla 1)
Límite de detección: valor que divide la muestra en no detectables y detectables no cuantificables.

Límite de cuantificación: valor que divide la muestra en detectables no cuantificables y cuantificables.

Tabla $\mathbf{N}^{\circ} 1$ : Valores límite y categorías de la variable BPA

\begin{tabular}{cccc}
\hline $\begin{array}{c}\text { Concentración de } \\
\text { BPA }\end{array}$ & Definición conceptual & Variable \\
\hline$<0,8 \mathrm{ng} / \mathrm{ml}$ & No detectables & Ausencia de la sustancia & Categoría I \\
\hline \multicolumn{2}{c}{ Límite de detección $0,8 \mathrm{ng} / \mathrm{ml}$} & Categoría II \\
\hline de 0,8 a $2 \mathrm{ng} / \mathrm{ml}$ & $\begin{array}{l}\text { Detectable no } \\
\text { cuantificable }\end{array}$ & Presencia de BPA no cuantificable & \\
\hline & Límite de cuantificación $2 \mathrm{ng} / \mathrm{ml}$ & Categoría III \\
\hline & Cuantificable & $\begin{array}{c}\text { Presencia de BPA pasible de ser } \\
\text { cuantificada }\end{array}$ & \\
\hline
\end{tabular}

Se calculó el porcentaje de muestras para cada una de las categorías y el porcentaje que resulta de la suma de muestras con BPA detectables no cuantificables y cuantificables, (categorías II y III). Todos los porcentajes se expresaron con su IC95\%.

Para las muestras con BPA cuantificable se obtuvo una distribución asimétrica, por lo que se calcularon medidas de resumen paramétricas y no paramétricas.

Método de muestreo: El tamaño muestral calculado fue de 111 mujeres, esperando encontrar un $90 \%$ de gestantes con presencia de BPA en orina ${ }^{(2,28)}$ con un error de 0,5 y una precisión de $+/-5 \%$. Previendo una posible pérdida del $40 \%$ se calculó un total de 150 mujeres. Se incluyeron de manera consecutiva a todas las pacientes que se realizaron la ecografía de translucencia nucal durante el período de estudio y que cumplían con los criterios de inclusión.

Análisis estadístico: Se realizó un análisis descriptivo de la población: edad, semana gestacional (al momento de la toma de muestra), nivel educativo y ocupación. Se describieron los hábitos de las mujeres asociados a la exposición de BPA. Se determinó el porcentaje (IC 95\%) de muestras con BPA en orina de embarazadas. La mediana de los valores cuantificables de BPA urinario se expresó en $\mathrm{ng} / \mathrm{ml}$ y $\mathrm{ng} / \mathrm{mg}$ de creatinina con su desvío estándar y rango intercuartilo.

El análisis estadístico se realizó con Epi Info versión 7.

Consideraciones éticas: El protocolo fue aprobado por el Comité de Ética del Hospital Italiano de Buenos Aires (CEPI). No existieron conflictos de intereses.

\section{Resultados}

Se contactaron 220 mujeres en sala de espera, de las cuales aceptaron participar 157 y se obtuvieron 149 muestras en total. El $98 \%$ de las participantes vivían en CABA o en el $1^{\circ}$ o $2^{\circ}$ cordón del Conurbano Bonaerense.

En la Tabla 2 se presenta el perfil de mujeres incluidas en el estudio, en el que se puede observar un alto porcentaje de mujeres que trabajan y un elevado nivel educativo.

Tabla $\mathbf{N}^{\circ}$ 2: Perfil de las embarazadas asistidas en el Hospital Italiano de Buenos Aires (n=149).

\begin{tabular}{|c|c|}
\hline Variable & \\
\hline Edad en años, media (DE) & $32,1(5,3)$ \\
\hline Edad gestacional en semanas, media (DE) & $12,3(1,1)$ \\
\hline Trabajadoras activas ${ }^{1}, \%$ (IC95\%) & $81(73,9-86,9)$ \\
\hline Nivel de instrucción universitario y posgrado ${ }^{2}, \%$ (IC95\%) & $83,7(76,7-89,1)$ \\
\hline Residencia en Ciudad Autónoma de Buenos Aires, \% (IC95\%) & $64(55,4-71,9)$ \\
\hline
\end{tabular}

En la tabla 3 se muestran los hábitos de las embarazadas que fueron incluidas en el estudio, pudiéndose observar un alto porcentaje de mujeres que utilizaban recipientes plásticos o envases con BPA. 
Tabla $\mathrm{N}^{\circ}$ 3: Hábitos frecuentes de las embarazadas asociados a la exposición a algún tipo de plástico o envase con BPA.

\begin{tabular}{llc}
\hline Variable & n & \% (IC95\%) \\
\hline Uso de conserva enlatada 2-3 veces al mes & 62 & $40,5(32,7-48,7)$ \\
Consumo de agua en recipiente de plástico (embotellado y/o dispenser)* & 67 & $45,0(36,9-53,3)$ \\
Uso frecuente de recipientes plásticos en heladera* & 71 & $47,6(39,5-55,9)$ \\
Uso de recipientes plásticos en microondas* & 74 & $48,1(39,9-56,2)$ \\
Trabajo relacionado con la industria del plástico & 6 & $3,9(1,4-8,3)$ \\
\hline
\end{tabular}

*Uso/Consumo habitual

Se encontró un porcentaje de muestras con BPA cuantificable en orina de embarazadas del $66,4 \%$ (IC95\% 58,2-74,0).

El porcentaje de muestras con presencia de BPA en orina de embarazadas asciende a $87,2 \%$ al incluir tanto las muestras con valores cuantificables como aquellas con valores detectables no cuantificables (Tabla 3). La mediana de BPA en las muestras cuantificables fue de $4.8 \mathrm{ng} / \mathrm{ml}$ con un rango de 2,1 a $30,1 \mathrm{ng} / \mathrm{ml}$.
Luego de realizar el ajuste de creatinina el valor de la mediana fue de $4,3 \mathrm{ng} / \mathrm{mg}$ de creatinina con un rango de 2,1 a 41,6. En 3 muestras de orina se encontraron concentraciones con valores superiores al percentilo 97 (20,3; 24,7 y 41,6 $\mathrm{ng} / \mathrm{mg}$ de creatinina respectivamente).

Tabla $N^{\circ}$ 4: Cuantificación de BPA en orina de embarazadas de Ciudad Autónoma de Buenos Aires

\begin{tabular}{|c|c|c|c|c|c|c|c|c|}
\hline $\begin{array}{l}\text { Presencia de } \\
\text { BPA en orina }\end{array}$ & $\mathbf{n}$ & \multicolumn{2}{|c|}{ Frecuencia relativa } & Mediana & $\begin{array}{c}\text { Rango } \\
\text { intercuartilo }\end{array}$ & Rango & Media & DE \\
\hline Total & 149 & \multicolumn{2}{|c|}{$\%(\mathrm{IC} 95 \%)$} & \multicolumn{5}{|c|}{ ng/mg de creatinina } \\
\hline Cuantificable & 99 & $66,4(58,2-74)$ & \multirow{2}{*}{$87,2(80,8-92,1)$} & 4,3 & $3,2-6,7$ & $2,1-41,6$ & 5,7 & 5 \\
\hline $\begin{array}{l}\text { Detectable no } \\
\text { cuantificable }\end{array}$ & 31 & $20,8(14,6-28,2)$ & & & & & & \\
\hline No detectable & 19 & $12,8(7,9-19,2)$ & $12,8(7,9-19,2)$ & & & & & \\
\hline
\end{tabular}

\section{Discusión}

Los resultados hallados en este estudio muestran que la población de mujeres de nuestro estudio, en su primer trimestre de embarazo, presenta BPA en orina.

El $87 \%$ de las mujeres de este estudio presentaron BPA en su orina, lo que implica que esta sustancia exógena derivada del plástico es absorbida, circula en el organismo y se elimina por orina constantemente en el primer trimestre de embarazo. Este valor es similar al reportado en la bibliografía revisada donde alcanza el $90 \%{ }^{(25)}$.

No se han encontrado hasta el momento publicaciones en nuestro país que informen concentraciones de BPA en orina. Si bien la muestra de este estudio representa a mujeres embarazadas de un plan de medicina prepaga del Área Metropolitana de la Ciudad Autónoma Buenos Aires, con alto nivel educativo y laboralmente activas, es evidente que la exposición al plástico en nuestra sociedad, es poblacional y no se encuentra determinada por diferencias socioeconómicas entre las personas ${ }^{(29)}$, por lo que estos resultados podrían ser considerados con validez externa para representar los niveles de orina de las embarazadas de nuestra comunidad. La literatura advierte el riesgo de contaminación de las muestras con BPA presentes en los materiales plásticos comunes en el laboratorio analítico. Esto fue estudiado, evaluado y documentado como parámetros de validación de la metodología bioanalítica empleada, la misma fue publicada por el laboratorio del HIBA $^{(27)}$

Los valores de BPA encontrados en orina de las mujeres embarazadas del HIBA son más altos que los reportados en otros estudios que muestran medianas de $2,2 \mathrm{ng} / \mathrm{ml}$ y $2,4 \mathrm{ng} / \mathrm{ml}$ ajustados por $\mathrm{mg}$ de creatinina ${ }^{(25,30)}$.

Una razón que podría explicar la diferencia en la concentración encontrada, es que las poblaciones estudiadas presentan hábitos y legislaciones diferentes respecto a la del presente estudio, algunos países, como China y Japón, han incorporado el BPA como un elemento regulado en los estándares de calidad del agua potable a diferencia de Argentina en el que solo está regulado el uso de BPA en mamaderas ${ }^{(31)}$.
Los valores límites que definen a las muestras como detectables o cuantificables en los diferentes estudios encontrados son menores $(0,4 \mathrm{ng} / \mathrm{ml})$ que los reportados en nuestra investigación. Esto se explica debido a que en las diferentes publicaciones citadas ${ }^{(25,30)}$, utilizan distintas metodologías para procesar las muestras siendo estas UPLC (Ultra Performance Liquid Chromatography) y MSMS (Tándem Mass Spectrometry). En el presente trabajo se utilizó el método validado GC MS (Gas chromatography-mass spectrometry ${ }^{(27)}$.

Esta diferencia en la metodología de medición de laboratorio podría explicar en parte las diferencias encontradas en la media y la mediana en nuestro estudio con respecto a la reportada en la literatura, así como podría estar subestimando la proporción de BPA encontrada.

En el presente trabajo también fueron analizados los hábitos de las embarazadas que podrían generar exposición a BPA (uso frecuente de recipientes de plástico, etc). El $100 \%$ de las embarazadas presentó al menos un hábito asociado al consumo de BPA. El uso de las diferentes fuentes de exposición se consideraron determinantes socio-culturales al estar arraigados culturalmente, lo que determina que la frecuencia de dichos hábitos fue muy alta entre las participantes. Por este motivo no se realizaron comparaciones de subgrupos (alto y bajo nivel de BPA). Epidemiológicamente se considera que las exposiciones que actúan a nivel poblacional (toda la población similarmente expuesta) invalidan las comparaciones a nivel individual (entre subgrupos) porque la variable de exposición se encontrará distribuida de manera similar entre los que presentan o no el resultado a medir, sesgando hacia la hipótesis nula ${ }^{(32)}$. Esto explica, además, la dificultad de contar con un grupo de referencia libre del factor de exposición con el cual establecer la relación del riesgo.

Otro motivo por el cual resulta difícil evidenciar los efectos de los $D E s^{(11)}$ es debido a que los efectos patológicos pueden manifestarse en ausencia evidente del factor de exposición o en un lapso de tiempo alejado del mismo. Si bien la toxicología clásica considera que el efecto de una sustancia está directamente relacionado con la dosis, los efectos de los DEs no cumplen esta regla pudiendo 
manifestarse a dosis mínima. Los resultados de este trabajo dejan abierta la pregunta sobre el potencial efecto tóxico frente a una exposición baja y constante durante la formación fetal. Sin embargo, este estudio brinda información basal para la cohorte de nacimientos seguida en el HIBA.

Al momento de la realización de este estudio la legislación vigente acerca de BPA no era uniforme y no existía consenso mundial en cuanto a la legislación que regula el uso del BPA en los plásticos. La Unión Europea prohibió el uso de BPA como aditivo en plásticos de mamaderas ${ }^{(31)}$ y estableció que existiría un límite de migración específica, es decir, una cantidad máxima de BPA permitida en los alimentos que no implicaría un riesgo para la salud, siendo esta 0,6 $\mathrm{mg} / \mathrm{kg}$ de alimento/día. Otros países como Francia, Suecia y Dinamarca, ampliaron el margen de prohibición a los recipientes utilizados para alimentos $^{(31)}$ (Ley No 2012-1442, 2012).

En Argentina, en el año 2012, la Administración Nacional de Medicamentos, Alimentos y Tecnología Médica (ANMAT) prohibió la fabricación, importación y comercialización de mamaderas que contengan BPA (Disposición ANMAT, Ministerio de Salud N 1207 , 2012).

\section{Conclusión}

El BPA en orina de embarazadas es una medida indirecta de exposición fetal y este estudio muestra que, al igual que en otros países, en un sector de la población de Argentina, la exposición es generalizada durante el proceso de formación embrionaria.

Las bajas concentraciones de BPA halladas en un alto porcentaje de las embarazadas deben ser interpretadas bajo el nuevo marco conceptual que apoya la toxicidad crónica a bajas dosis para evitar considerarlas irrelevantes hasta tanto se determine el efecto a largo plazo en nuevos estudios sobre los descendientes.

Conflictos de interés: Los autores no tienen conflictos de interés respecto de esta investigación.

Limitaciones de responsabilidad: la institución responsable de los datos incluidos en la investigación es el Hospital Italiano de Buenos Aires. No existe una política actual sobre Intercambio de datos en el Hospital Italiano de Buenos Aires. Los datos y códigos de computación pueden ser solicitados para el autor correspondiente que luego presentará la petición a la Junta de Ética Institucional, quien decidirá qué información puede estar disponible.

Fuentes de financiación: El presente trabajo de investigación fue realizado con el apoyo del programa de becas "Ramón Carrillo-Arturo Oñativia", categoría individual, otorgada por el Ministerio de Salud de la Nación Argentina, a través de la Comisión Nacional Salud Investiga. Año 2013.

\section{Bibliografía}

1. Olea N, Molina MJ, Garcia-Martin M, Olea-Serrano MF. Modern agricultural practices: The human price. J Toxicol Environ Health B Crit Rev. 1996; 5(4): 455-474.

2. Center for Disease control and prevention, National Health and nutrition Examination Survey: lab methods 2003-2004. 2008.

3. Vandenberg LN, Colborn T, Hayes TB, Heindel JJ, Jacobs Jr $D R$, Lee $D H$ et al. Hormones and endocrine-disrupting chemicals: low-dose effects and nonmonotonic dose responses. Endocr Rev. 2012 Jun; 33(3): 378-455.

4. Programa de las Naciones Unidas para el Medio Ambiente. Convenio de Estocolmo sobre Contaminantes Orgánicos Persistentes. 2005.

5. Mileva G, Baker SL, Konkle ATM, Bielajew C. Bisphenol-A: epigenetic reprogramming and effects on reproduction and behavior. Int J Environ Res Public Health. 2014; 11(7): 75377561.

6. Vandenberg LN, Chahoud I, Heindel JJ, Padmanabhan V, Paumgartten FJR, Schoenfelder G. Urinary, circulating, and tissue biomonitoring studies indicate widespread exposure to bisphenol A. Environ Health Perspect. 2010 Mar 24; 118(8): 1055.

7. Lorber M, Schecter A, Paepke O, Shropshire W, Christensen K, Birnbaum L. Exposure assessment of adult intake of bisphenol
A (BPA) with emphasis on canned food dietary exposures. Environ Int, Apr 2015; 77: 55-62.

8. González GL, Tejera Pérez, Cintia Hernández-Sánchez, Carmen Rubio Armendáriz, Consuelo Revert Gironés, Angel Gutiérrez Fernández,González, G.L., et al., Toxicidad del Bisfenol A (BPA): migración desde los envases a los alimentos. Aula de la farmacia: revista profesional de formación continuada. 2011; 7(87): 58-65.

9. Shimizu M, Ohta K, Matsumoto Y, Fukuoka M, Ohno Y, Ozawa $S$. Sulfation of bisphenol $A$ abolished its estrogenicity based on proliferation and gene expression in human breast cancer MCF7 cells. Toxicol In Vitro. 2002 oct; 16(5): p. 549-56.

10. Balakrishnan B, Henare $K$, Thorstensen EB, Ponnampalam AP, Mitchell MD. Transfer of bisphenol $A$ across the human placenta. Am J Obstet Gynecol. 2010 Apr; 202(4): 393 e1-7.

11. Fernández MF, Olea N. Endocrine disruptors. Is there sufficient evidence to act? Gac Sanit. 2014 Mar-Apr;28(2):93-5.

12. Falero Morejón A, Pérez $C$, Luna B, Fonseca M. Impacto de los disruptores endocrinos en la salud y el medio ambiente. Revista CENIC. Ciencias Biológicas. 2005; 36.

13. López-Espinosa MJ. Exposición materno-infantil vía placentaria a compuestos químicos medioambientales con actividad hormonal. Editorial de la Universidad de Granada. 2006.

14. Juan-García A, Gallego C, Font G. Toxicidad del Bisfenol A: Revisión. Revista de Toxicología. 2015; 32 (2): 144-160.

15. Singh S, Li SS. Epigenetic effects of environmental chemicals bisphenol A and phthalates. Int J Mol Sci. 2012; 13(8): 1014353.

16. Muñoz-de-Toro M, Markey CM, Wadia PR, Luque EH, Rubin $B S$, Sonnenschein $C$, et al. Perinatal exposure to bisphenol- $A$ alters peripubertal mammary gland development in mice. Endocrinology. 2005 Sep;146(9):4138-47.

17. Diamanti-Kandarakis E, Palioura E, Kandarakis SA, Koutsilieris $M$. The impact of endocrine disruptors on endocrine targets. Horm Metab Res. 2010 Jul;42(8):543-52.

18. Goldman L, Falk H, Landrigan PJ, Balk SJ, Reigart JR, Etzel $R A$. Environmental pediatrics and its impact on government health policy...Pediatrics. 2004 Apr;113(4 Suppl):1146-57.

19. Cabaton NJ, Wadia PR, Rubin BS, Zalko D, Schaeberle CM, Askenase $\mathrm{MH}$ et al. Perinatal exposure to environmentally relevant levels of bisphenol $A$ decreases fertility and fecundity in CD-1 mice. Environ Health Perspect. 2011 Apr;119(4):547-52.

20. Miao M, Yuan W, He Y, Zhou Z, Wang J, Gao E et al. In utero exposure to bisphenol-A and anogenital distance of male offspring..Birth Defects Res A Clin Mol Teratol. 2011 Oct; $91(10): 867-72$.

21. Gill WB, Schumacher GF, Bibbo M, Straus FH 2nd, Schoenberg $H W$. Association of diethylstilbestrol exposure in utero with cryptorchidism, testicular hypoplasia and semen abnormalities. $J$ Urol. 1979 Jul;122(1):36-9.

22. Argemi F, Cianni N, Porta A. Disrupción endocrina: perspectivas ambientales y salud pública. Acta bioquím. clín. latinoam. 2005 Jun; 39 (3): 291-300.

23. Caserta D, Bordi G, Ciardo F, Marci R, La Rocca C, Tait $S$ et al. The influence of endocrine disruptors in a selected population of infertile women. Gynecol Endocrinol. 2013 May;29(5):444-7.

24. Guillette Jr LJ, Crain DA. Endocrine disrupting contaminants: an evolutionary perspective. 1st ed. Philadelphia: Francis and Taylor Inc; 2000.

25. Calafat AM, Ye X, Wong LY, Reidy JA, Needham LL.Exposure of the U.S. population to bisphenol $A$ and 4-tertiary-octylphenol: 2003-2004. Environ Health Perspect. 2008 Jan;116(1):39-44.

26. Ye X1, Pierik FH, Hauser R, Duty S, Angerer J, Park MM, Burdorf $A$ et al. Urinary metabolite concentrations of organophosphorous pesticides, bisphenol $A$, and phthalates among pregnant women in Rotterdam, the Netherlands: the Generation R study. Environ Res. 2008 Oct;108(2):260-7.

27. Cases GG, Uicich RE, Kandel Gambarte $P$, Vázquez M L, Moix $C F$, Ferloni $A$, et al. Determinación de los Niveles de Bisfenol A en Orina de Mujeres Embarazadas por Cromatografía Gaseosa - Espectrometría de Masa. Acta Bioquímica Clínica Latinoamericana. Forthcoming 2019. 
28. Lang IA, Galloway TS, Scarlett A, Henley WE, Depledge M, Wallace $R B$ et al. Association of urinary bisphenol $A$ concentration with medical disorders and laboratory abnormalities in adults. JAMA. 2008 Sep 17;300(11):1303-10.

29. Miao M, Wang Z, Liu X, Liang H, Zhou Z, Tan H et al. Urinary bisphenol $A$ and pubertal development in Chinese school-aged girls: a cross-sectional study. Environ Health. 2017 Jul 27:16(1):80.

30. Casas L, Fernández MF, Llop S, Guxens M, Ballester F, Olea N et al. Urinary concentrations of phthalates and phenols in a population of Spanish pregnant women and children. Environ Int. $2011 \mathrm{Jul} ; 37(5): 858-66$.

31. Yuan SF, Liu ZH, Lian $H X$, Yang $C$, Lin $Q$, Yin $H$ et al. Simultaneous determination of estrogenic odorant alkylphenols, chlorophenols, and their derivatives in water using online headspace solid phase microextraction coupled with gas chromatography-mass spectrometry. Environ Sci Pollut Res Int. 2016 Oct;23(19):19116-25.

32. Rose, G. (2001). Sick individuals and sick populations. International Journal of Epidemiology. 2001 Jun;30(3): 427-432. 